Document downloaded from:

http://hdl.handle.net/10251/52186

This paper must be cited as:

Benítez López, J.; Delgado Galván, XV.; Izquierdo Sebastián, J.; Pérez García, R. (2012). Improving consistency in AHP decision-making processes. Applied Mathematics and Computation. 219(5):2432-2441. doi:10.1016/j.amc.2012.08.079.

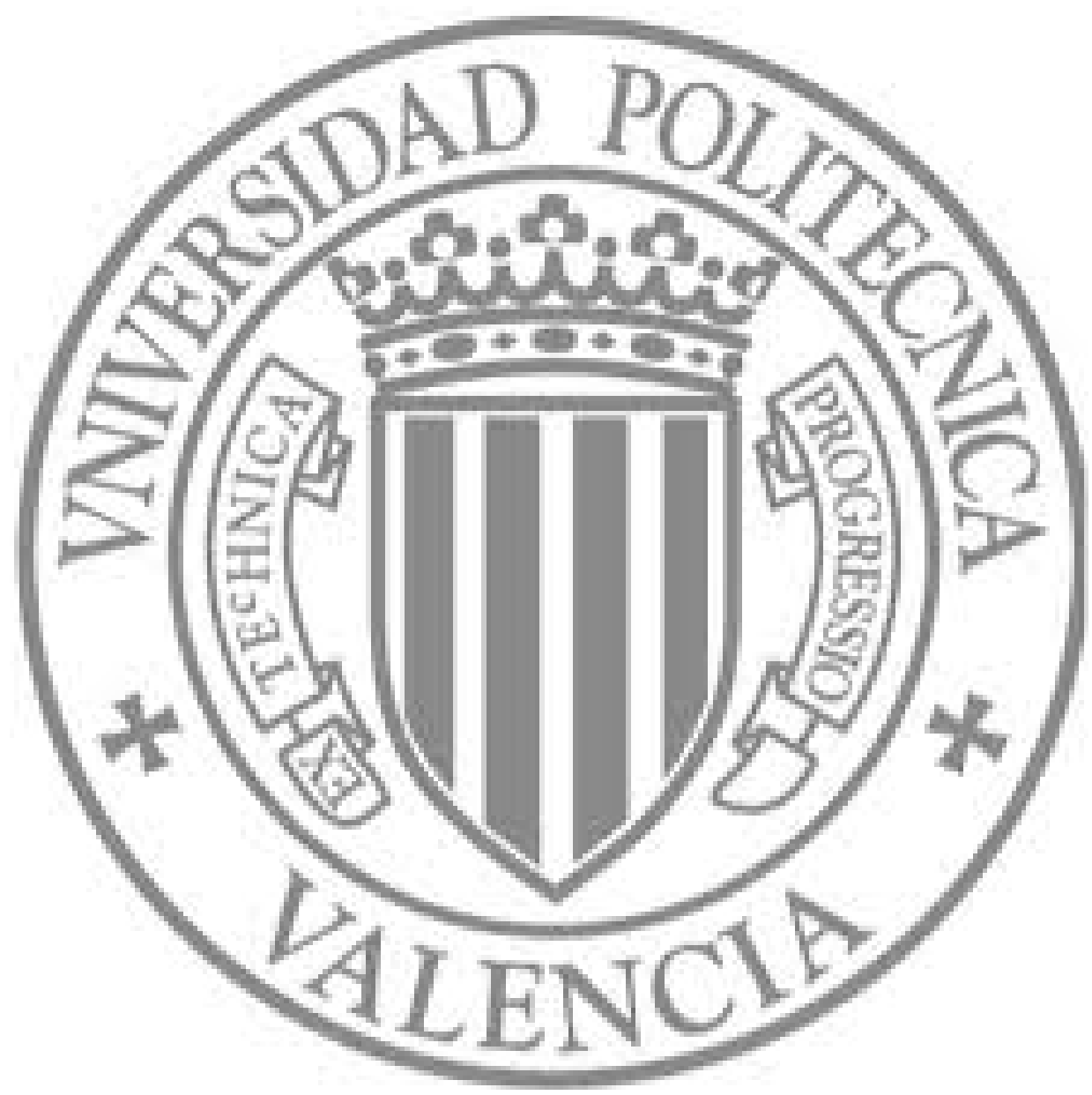

The final publication is available at

http://dx.doi.org/10.1016/j.amc.2012.08.079

Copyright Elsevier 


\title{
Improving Consistency in AHP Decision-Making Processes
}

\author{
J. Benítez, X. Delgado-Galván, J. Izquierdo, R. Pérez-García \\ Fluing-I.M.M., Universitat Politècnica de València, \\ Camino de Vera s/n, 46022 Valencia, Spain
}

\begin{abstract}
Decision making in engineering is becoming increasingly complex due to the large number of alternatives and multiple conflicting goals. Powerful decisionsupport expert systems powered by suitable software are increasingly necessary. In this paper, the multiple attribute decision method known as analytical hierarchy process (AHP), which uses pairwise comparisons with numerical judgments, is considered. Since judgments may lack a minimum level of consistency, mechanisms to improve consistency are necessary. A method to achieve consistency through optimisation is described in this paper. This method has the major advantage of depending on just $n$ decision variables - the number of compared elements - and so is less computationally expensive than other optimisation methods, and can be easily implemented in virtually any existing computer environment. The proposed approach is exemplified by considering a simplified version of one of the most important problems faced by water supply managers, namely, the minimisation of water loss.
\end{abstract}

Keywords: analytic hierarchy process, consistent matrices, decision making, optimisation, water distribution systems, leaks.

\section{Introduction}

A variety of powerful tools have emerged in recent decades to help decision makers understand and analyse various types of decisions. Decisions are usually dynamic in

\footnotetext{
${ }^{*}$ Corresponding author. E-mail address: jizquier@upv.es; Tel.: +34 628028804
} 
nature and arise in every type of application - including engineering, medical management, sports, and emergency situations. This paper considers one of the main challenges that water supply managers face: the minimisation of water loss $[1,2,3,4,5]$. Vast sums are devoted annually to this aim worldwide.

There are many reasons why today's decisions are becoming increasingly complex: more intangible elements, heightened uncertainty and subjectivity, shorter deadlines, greater pressure, rapidly changing conditions, and higher risks. In this paper, focus is placed on the various types of system attributes that permeate all the current multicriteria decision making processes.

There are two types of system attributes that need to be considered in decisionmaking: objective and subjective. Objective attributes are measured and defined in numerical terms. For example, engineering attributes, such as pipe capacity, load conditions, pressure head, demands, and energy expenses; or cost attributes, such as acquisition and installation costs for various elements, maintenance costs, and penalties for lack of service. Subjective attributes may be considered as qualitative and include damage to goods and properties, consequences of service disruption, increases in vehicle driving time, congested traffic delays, and the cost of $\mathrm{CO}_{2}$ emissions derived from energy consumption. These attributes cannot be precisely and numerically measured by the decision maker.

Nevertheless, decision makers need to be concerned not only with the tangible and quantitative factors, such as the cost in engineering selection problems, but also with the intangible and qualitative factors, such as environmental and social impacts [6]. Let us refer back to the problem under examination. By considering an exclusively economic point of view, investment in minimising water loss is usually balanced by the benefits derived from the use of the recovered water. Nevertheless, this scheme does not reflect the whole dimension of the benefit-earning capacity of repairing leaks. The associated benefits may include more aspects than just the economic value of the recovered water. In this paper, an economic assessment approach that includes other costs caused by leaks and the benefits derived from their control is considered. These are mainly environmental and social costs and benefits, which are called externalities from an economic point of view. Their inclusion renders the assessment of leaks more 
realistic, but raises important problems about how such costs and benefits should be considered. The main problem derives from the fact that comparisons with regard to certain properties will only work for properties with well-defined scales of measurement. Nevertheless, direct comparisons are necessary to establish measurements for intangible properties that have no scales of measurement.

The analytical hierarchy process (AHP) [7] is a multiple attribute decision method that uses structured pairwise comparisons with numerical judgments from an absolute scale of numbers. AHP has been applied in several areas, such as logistics, manufacturing, government, and education [8].

The fundamentals of AHP, including its hierarchical, multi-level structure with goals, criteria, and alternatives, the way judgment is compiled into positive reciprocal matrices, the estimation of the relative weights of the decision elements, the use of prioritisation techniques, and the way in which aggregation is performed to obtain a final composite vector of priorities can be found in any handbook and many papers about the subject (see, for instance [7, 9]). Ultimately, the decision-making problem is discrete and involves selecting the best alternatives from a finite set of feasible choices based on the evaluation of each against a given set of criteria.

When using judgment to estimate dominance in making comparisons between two alternatives - and especially for intangibles - instead of using numbers from a scale, a single number drawn from a fundamental scale of absolute numbers is assigned. Judgment must be based on knowledge, that is to say, on data. One method to collect data is by directly interviewing experts. This data can be supplemented with contingent valuation methods [10]; or by the participation of an expert panel [11]; or by applying the Delphi technique (a systematic and interactive forecasting method that relies on a panel of experts for forecasting) [12]. The person coordinating the Delphi method is known as a facilitator, and facilitates the responses of the panel of experts.

Pairwise comparisons are quantified using a scale. There are several approaches in developing such scales [13]. In this paper, we consider a nine-point scale developed by Saaty $[9,14]$, with the possibility of including intermediate numerical (decimal) values in the scale to model hesitation between two adjacent judgments [15]. To extract priority vectors from the comparison matrices, among the many existing methods 
$[16,17,18,19]$, the eigenvector method, which was first proposed by Saaty in his seminal paper [9] in 1977, is used in this paper.

A comparison matrix, $A$, exhibits two basic properties, namely homogeneity $\left(a_{i j}=\right.$ 1 , if elements $i$ and $j$ are considered equally important; in particular $a_{i i}=1$ for every $i)$ and reciprocity $\left(a_{j i}=1 / a_{i j}\right.$ for all $\left.i, j\right)$. Besides these two properties, a third property, that of consistency, should theoretically be desirable for a comparison matrix. A positive $n \times n$ matrix is consistent if $a_{i j} a_{j k}=a_{i k}$, for $i, j, k=1, \ldots, n$. Consistency expresses the coherence that may exist between judgments about the elements of a set. Since preferences are expressed in a subjective manner it is reasonable (and, arguably, even desirable) for some kind of incoherence to exist. When dealing with intangibles, judgments are rarely consistent unless they are forced in some artificial manner.

$A$ is not generally consistent because it only contains the comparison values obtained through numerical judgment. For most problems, estimates of these values by an expert are assumed to be small perturbations of the 'right' values. This implies small perturbation of the eigenvalues (see, for instance, $[20]$ ).

The next problem to solve is the eigenvalue problem $A \mathbf{w}=\lambda_{\max } \mathbf{w}$, where $\lambda_{\max }$ is, according to the Perron-Frobenius theory (see, for example, [21]), the unique largest eigenvalue of $A$, which also gives the so-called Perron eigenvector, that is an estimate of $Z$, the priority vector. A decision about the consistency level of a matrix is now crucial.

Although various measurements of inconsistency can be developed, the measurement proposed by Saaty [7] is used in this paper. The intrinsic consistency threshold developed by Monsuur [22] is also used.

In [18] Finan and Hurley state that additional artificial manipulation to increase consistency will on average improve the reliability of an analysis. Several alternatives to improve consistency, mostly based on various optimisation techniques, have been proposed in the literature. In this paper, a solution based on the minimisation of the distance between two matrices that uses a truly reduced number of decision variables is proposed. As a consequence, the process may be accomplished with no computational burden at all.

This paper is a revised and extended version of a conference paper [23]. The re- 
mainder of this paper is structured as follows. In the following section, the notation and necessary elements used later - a reduced version of a number of results included in [23] - is concisely presented. Based on these properties, in Section 3 an optimisation technique (the main contribution in [23]) is developed that produces the closest consistent matrix to a given positive reciprocal matrix, and which involves only $n$ decision variables ( $n$ being the order of the matrix) and just one constraint, in stark contrast to other optimisation methods $[6,24,25]$ that consider $O\left(n^{2}\right)$ decision variables and many constraints (Section 3 being a significant expansion of [23]). A simple decision-support expert system implementing various prioritising methods, in particular the method presented in this paper, has also been added (Section 4). Finally, these results are applied to a comparison between two alternatives in water supply management, namely, active leakage control and passive leakage control. The paper closes with enriched conclusions.

\section{Notation and review of the properties of consistent ma- trices}

$M_{n, m}$ will hereinafter denote the set of $n \times m$ real matrices, and $M_{n, m}^{+}$will denote the subset of $M_{n, m}$ composed of positive matrices. It will be assumed that the elements of $\mathbb{R}^{n}$ are column vectors, i.e., $\mathbb{R}^{n}$ is identified with $M_{n, 1}$. For a given $A \in M_{n, m}$, let us write $[A]_{i j}$ the $(i, j)$ entry of the matrix $A$. The superscript $T$ denotes the matrix transposition.

The mapping $J: M_{n, m}^{+} \rightarrow M_{n, m}^{+}$defined by $[J(A)]_{i j}=1 /[A]_{i j}$ will play an important role in the sequel. The following facts are evident : If $A \in M_{n, m}^{+}$and $\lambda>0$, then $J^{2}(A)=A$ and $J(\lambda A)=\lambda^{-1} J(A)$. If, in addition, $\mathbf{x} \in M_{n, 1}^{+}$, then $\mathbf{x}^{T} J(\mathbf{x})=J(\mathbf{x})^{T} \mathbf{x}=$ $n$. Obviously, a matrix $A \in M_{n, 1}^{+}$is reciprocal if and only if $J(A)=A^{T}$.

The next result [23] gathers well knows equivalent facts (see [7, 26], among others) and enables the definition of consistent matrices.

Theorem 1. Let $A \in M_{n, n}^{+}$. If $a_{i j}=[A]_{i, j}$, then following statements are equivalent.

1. There exists $\mathbf{x} \in M_{n, 1}^{+}$such that $A=J(\mathbf{x}) \mathbf{x}^{T}$. 
2. There exists $\mathbf{w}=\left[\begin{array}{lll}w_{1} & \ldots & w_{n}\end{array}\right]^{T} \in M_{n, 1}^{+}$such that $a_{i j}=w_{i} / w_{j}$ for all $i, j \in$ $\{1, \ldots, n\}$.

3. $a_{i j} a_{j i}=1$ and $a_{i j} a_{j k}=a_{i k}$ hold for all $i, j, k \in\{1, \ldots, n\}$.

It is worthwhile providing an interpretation of this theorem. Statement 3 corresponds to the definition of consistency given in Section 1. Statement 1 provides the main tool in this paper for developing the optimisation process described in Section 3. Finally, the components of vector $\mathbf{w}$ given by Statement 2 may be considered as absolute values for any of the elements involved in the process. If such values are known, the comparisons are straightforward: $a_{i j}=\mathbf{w}_{i} / \mathbf{w}_{j}$ for $i, j=1, \ldots, n$. In general, however, such absolute values are unknown. Specifically, the aim of AHP is to assign to each of the $n$ elements under comparison, priority values $\mathbf{w}_{i}, i=1, \ldots, n$, that reflect the emitted judgments. If judgments are consistent, the relations between the judgments, $a_{i j}$, and the values, $\mathbf{w}_{i}$, are $a_{i j}=\mathbf{w}_{i} / \mathbf{w}_{j}, i, j=1, \ldots, n$, and $A$ is consistent.

Item 3 of Theorem 1 shows that any consistent matrix is reciprocal. Even though the converse is false in general, however, it is simple to prove that any reciprocal matrix of order 2 is consistent.

It is worthwhile noting that for a given consistent matrix $A=\left(a_{i j}\right)$, the vector $\mathbf{x}=\left[\begin{array}{lll}a_{11} & \cdots & a_{1 n}\end{array}\right]^{T}$ satisfies $A=J(\mathbf{x}) \mathbf{x}^{T}$. It is also well known that the rank of any matrix of the form $\mathbf{u v}^{T}$ (where $\mathbf{u}, \mathbf{v} \in M_{n, 1}$ are nonzero vectors) is 1 , hence the rank of any consistent matrix is one. Furthermore, for a consistent matrix $A$ written as in item 1 of Theorem 1 one has $A J(\mathbf{x})=J(\mathbf{x}) \mathbf{x}^{T} J(\mathbf{x})=n J(\mathbf{x})$, which proves the very well known fact that $n$ is an eigenvalue of any consistent matrix $A=J(\mathbf{x}) \mathbf{x}^{T}$ of order $n$ and $J(\mathbf{x})$ is an eigenvector of $A$ associated with $n$. Moreover, it is known (see, for example, [21, Exercise 7.2.13]) that any matrix of the form $\mathbf{u v}^{T}$ (where $\mathbf{u}, \mathbf{v} \in M_{n, 1}$ are nonzero) is diagonalizable if and only if $\mathbf{u}^{T} \mathbf{v} \neq 0$. Therefore, any consistent matrix is diagonalisable. Thus, taking into account that the rank of $A$ is 1 , any eigenvector of $A$ associated with $n$ is a scalar multiple of $J(\mathbf{x})$.

If $\lambda>0$ and $\mathbf{x} \in M_{n, 1}^{+}$, then obviously $J(\lambda \mathbf{x})(\lambda \mathbf{x})^{T}=J(\mathbf{x}) \mathbf{x}^{T}$. Does a type of converse hold? If $\mathbf{x} \in M_{n, 1}^{+}$and $\mathbf{y} \in M_{n, 1}^{+}$satisfy $J(\mathbf{x}) \mathbf{x}^{T}=J(\mathbf{y}) \mathbf{y}^{T}$, then $x_{j} / x_{i}=y_{j} / y_{i}$ 
for all $i, j \in\{1, \ldots, n\}$. Hence $x_{j} / y_{j}$ does not depend on the index $j$, and thus, by denoting $\lambda=x_{j} / y_{j}$, one has $x_{i}=\lambda y_{i}$ for all $i \in\{1, \ldots, n\}$. Therefore, the following result holds.

Theorem 2. Let $\mathbf{x}, \mathbf{y} \in M_{n, 1}^{+}$such that $J(\mathbf{x}) \mathbf{x}^{T}=J(\mathbf{y}) \mathbf{y}^{T}$. Then there exists $\lambda>0$ such that $\mathbf{x}=\lambda \mathbf{y}$.

From the Perron-Frobenius theory (see e.g., [21, Chapter 8]) it is known that there is $\lambda_{\max }$, an eigenvalue of $A$, such that $\lambda_{\max }>|\lambda|$ for any $\lambda$ eigenvalue of $A$ (the eigenvalue $\lambda_{\max }$ is called the Perron root of $A$ ). Saaty proved [7] that $\lambda_{\max } \geq n$, and the equality holds if and only if $A$ is consistent. At this point another criterion for a reciprocal matrix to be consistent may be given. It will be used in the next section to achieve consistency for a non-consistent matrix.

Theorem 3. Let $A$ be a reciprocal matrix. Then $A$ is consistent if and only if $\operatorname{rank}(A)=1$.

Proof: As stated before, any consistent matrix has rank one. Assume that $A$ is a reciprocal matrix whose rank is 1 . It is a simple textbook exercise that any matrix $A \in M_{n, n}$ having rank 1 can be written as $A=\mathbf{u v}^{T}$, where $\mathbf{u}, \mathbf{v} \in \mathbb{R}^{n}$ are nonzero. As $A$ is positive, it can be assumed that the vectors $\mathbf{u}$ and $\mathbf{v}$ are positive. From $A^{T}=J(A)$

$$
\mathbf{v} \mathbf{u}^{T}=J\left(\mathbf{u v}^{T}\right)=J(\mathbf{u}) J(\mathbf{v})^{T}
$$

is obtained. Pre-multiplying by $\mathbf{v}^{T}$ and transposing yield $\left(\mathbf{v}^{T} \mathbf{v}\right) \mathbf{u}=\left(\mathbf{v}^{T} J(\mathbf{u})\right) J(\mathbf{v})$. Taking into account from this point that $\mathbf{u}$ and $\mathbf{v}$ are positive, it is possible to write $\mathbf{u}=\lambda J(\mathbf{v})$ being $\lambda>0$. Using (1) produces $\lambda \mathbf{v} J(\mathbf{v})^{T}=J(\lambda J(\mathbf{v})) J\left(\mathbf{v}^{T}\right)=\lambda^{-1} \mathbf{v} J(\mathbf{v})^{T}$. From Theorem 1 and bearing in mind that $\mathbf{v} J(\mathbf{v})^{T} \neq 0$ it is found that $\lambda=\lambda^{-1}$. Using $\lambda>0$ leads to $\lambda=1$. Since $A=\mathbf{u v}^{T}$ and $\mathbf{u}=\lambda J(\mathbf{v})$, Theorem 1 finishes the proof.

\section{Achieving matrix consistency}

As stated above, the interest is in finding (approximate) solutions for the following problem: given $A \in M_{n, n}^{+}$, find a consistent matrix $B$ such that $A$ 'is close to' $B$.

Several alternatives, mostly based on various optimization techniques, have been proposed in the literature to help improve consistency. The weighted least squares [16] 
method tries to minimize the sum of errors of the differences between the judgments and their derived values. In [25] it is proposed a goal programming method that uses relative deviations to force changes in the values of the comparisons so that the target values differ as little as possible from the original values - while approximately taking homogeneity into account and preserving reciprocity and consistency. A slight modification of this method that reduces the number of decision variables and constraints is used in [6]. The logarithm least squares method has a long history and has been intensively studied by many authors (e.g. [17, 27, 28]), although it has been shown that is equivalent to the normalization of geometric means of rows (NGMR), which is easier since NGMR consists in multiplying the $n$ elements in each row and taking the $n$th root, and then normalising so that these numbers add up to unity [17, 29, 30]. Other methods include fuzzy programming [31] and enhanced goal programming [27]. In [24], a method is developed that uses linear programming (LP); the output is easy to understand, and sensitivity analysis can be performed using the LP standard theory. Even though not fitting strictly the form of an optimization problem, various other methods deserve being considered here. For example, Saaty [32] proposed a method based on perturbation theory to find the most inconsistent judgment in the matrix; this action could be followed by the determination of the range of values to which that judgment could be changed and whereby the inconsistency could be improved - and then asking the judge to consider changing the judgment to a plausible value within that range. The authors have recently developed a method [33] based on a linearization process [34] that follows an iterative feedback process to achieve an acceptable level of consistency while complying to some degree with expert preferences.

Despite the abundance of prioritisation methods, it becomes clear that those methods based on optimization perform, in general, better than other more direct methods. Also, as shown in [19], 'none of prioritization methods perform better than others in every inconsistent case', and 'the most appropriate prioritisation operator is in fact on a case-by-case basis'. The purpose of this section (and of the paper) is to provide another optimisation process that has the important advantage of depending of only $n$ decision variables - the number of compared elements. Thus, this process is simpler, less computationally expensive, and can be easily implemented either in a stand-alone 
piece of software using any optimisation library or integrated in a specific platform built using any of the existing computer environments, as the one presented below.

The concept of closeness between matrices is, of course, defined in terms of a matrix norm (see [21, Section 5.2]). This is the approach used in this paper, namely, the minimisation of a matrix norm using Theorem 1, which enables reducing the number of decision variables to just $n$, the order of the matrix. Thus, the main purpose of this section will be to study the following problem: given $A \in M_{n, n}^{+}$, find a consistent matrix $B$ such that $\|A-B\|$ 'is small'. The Frobenius matrix norm is proposed in view of its simplicity. Such a norm is defined as

$$
\|A\|_{\mathrm{F}}=\left(\sum_{i, j}[A]_{i j}^{2}\right)^{1 / 2}=\left[\operatorname{tr}\left(A^{T} A\right)\right]^{1 / 2}, \quad A \in M_{n, m} .
$$

Let us, then, formally write the posed problem as:

Problem 1. Let $A \in M_{n, n}^{+}$. Find $\mathbf{x} \in M_{n, 1}^{+}$such that

$$
\min _{\mathbf{y} \in M_{n, 1}^{+}}\left\|A-J(\mathbf{y}) \mathbf{y}^{T}\right\|_{\mathrm{F}}=\left\|A-J(\mathbf{x}) \mathbf{x}^{T}\right\|_{\mathrm{F}}
$$

Using the Frobenius matrix norm is equivalent to minimising the root mean square variance of the differences $a_{i j}-\mathbf{w}_{i} / \mathbf{w}_{j}$, which is one of the measurement methods propagating measurement priority vectors described in [19].

Observe that $J(\lambda \mathbf{x})(\lambda \mathbf{x})^{T}=J(\mathbf{x}) \mathbf{x}^{T}$ holds for any $\lambda>0$ and $\mathbf{x} \in M_{n, 1}^{+}$. Therefore, bearing in mind Theorem 2, Problem 1 can be 'normalised' to the following:

Problem 2. Let $A \in M_{n, n}^{+}$and $\|\cdot\|$ a norm in $\mathbb{R}^{n}$. Find $\mathbf{x} \in M_{n, 1}^{+}$such that $\|\mathbf{x}\|=1$ and

$$
\min _{\mathbf{y} \in M_{n, 1}^{+},\|\mathbf{y}\|=1}\left\|A-J(\mathbf{y}) \mathbf{y}^{T}\right\|_{\mathrm{F}}=\left\|A-J(\mathbf{x}) \mathbf{x}^{T}\right\|_{\mathrm{F}}
$$

It will be shown in the next result that the solution of Problem 2 does not depend on the considered norm in $\mathbb{R}^{n}$.

Theorem 4. Let $\|\cdot\|$ and $|\cdot|$ be two norms in $\mathbb{R}^{n}$. If $\mathbf{u}$ is a solution of the Problem 2 with respect to the norm $\|\cdot\|$, then $\mathbf{u} /|\mathbf{u}|$ is a solution to Problem 2 with respect to the norm $|\cdot|$. 
Proof: Define $\widehat{\mathbf{u}}=\mathbf{u} /|\mathbf{u}|$. To prove the theorem, it must be shown that

$$
\left\|A-J(\widehat{\mathbf{u}}) \widehat{\mathbf{u}}^{T}\right\|_{\mathrm{F}} \leq\left\|A-J(\mathbf{y}) \mathbf{y}^{T}\right\|_{\mathrm{F}} \quad \forall \mathbf{y} \in M_{n, 1}^{+} \text {such that }|\mathbf{y}|=1
$$

Pick any $\mathbf{y} \in M_{n, 1}^{+}$such that $|\mathbf{y}|=1$. Define $\widehat{\mathbf{y}}=\mathbf{y} /\|\mathbf{y}\|$. Since $\mathbf{u}$ is a solution to Problem 2, one has

$$
\left\|A-J(\mathbf{u}) \mathbf{u}^{T}\right\|_{\mathrm{F}} \leq\left\|A-J(\widehat{\mathbf{y}}) \widehat{\mathbf{y}}^{T}\right\|_{\mathrm{F}}
$$

Since $\widehat{\mathbf{u}}$ is a scalar multiple of $\mathbf{u}$, one has $J(\mathbf{u}) \mathbf{u}^{T}=J(\widehat{\mathbf{u}}) \widehat{\mathbf{u}}^{T}$. Similarly, $J(\mathbf{y}) \mathbf{y}^{T}=$ $J(\widehat{\mathbf{y}}) \widehat{\mathbf{y}}^{T}$ is obtained. Consequently, (3) enables (2) to be proven.

Hence any norm in $\mathbb{R}^{n}$ can be used to solve the minimisation Problem 2. Two norms in $\mathbb{R}^{n}$ will be proposed.

1. The 1-norm in $\mathbb{R}^{n}$ (i.e., $\|\mathbf{x}\|_{1}=\left|x_{1}\right|+\cdots+\left|x_{n}\right|$ ). This norm is proposed since for any $\mathbf{x} \in M_{n, 1}^{+}$, it is found that $\|\mathbf{x}\|_{1}=x_{1}+\cdots+x_{n}$, which is a very simple and differentiable expression.

2. The 2-norm (or Euclidean norm) in $\mathbb{R}^{n}$ (i.e., $\|\mathbf{x}\|_{2}=\left(x_{1}^{2}+\cdots+x_{n}^{2}\right)^{1 / 2}=$ $\left.\left(\mathbf{x}^{T} \mathbf{x}\right)^{1 / 2}\right)$. It will be noticed that $\|\cdot\|_{2}$ is differentiable (the origin is the unique point of non-differentiability and $\|\mathbf{0}\|_{2} \neq 1$ ). Although the expression of this norm is more complicated than the 1-norm, the Euclidean norm is also proposed in view of the Theorem 5 result shown below.

Theorem 5. Let $A \in M_{n, n}$ and $\mathbf{x} \in M_{n, 1}$. Then

$$
\left\|A-J(\mathbf{x}) \mathbf{x}^{T}\right\|_{\mathrm{F}}^{2}=\|A\|_{\mathrm{F}}^{2}+\|\mathbf{x}\|_{2}^{2}\|J(\mathbf{x})\|_{2}^{2}-2 J(\mathbf{x})^{T} A \mathbf{x}
$$

Proof: Before proving this theorem, notice that $\operatorname{tr}(X Y)=\operatorname{tr}(Y X)$ holds for any pair of matrices $X$ and $Y$ such that $X Y$ and $Y X$ are meaningful. Now,

$$
\begin{aligned}
{[A-} & \left.J(\mathbf{x}) \mathbf{x}^{T}\right]^{T}\left(A-J(\mathbf{x}) \mathbf{x}^{T}\right) \\
& =\left(A^{T}-\mathbf{x} J(\mathbf{x})^{T}\right)\left(A-J(\mathbf{x}) \mathbf{x}^{T}\right) \\
& =A^{T} A-\mathbf{x} J(\mathbf{x})^{T} A-A^{T} J(\mathbf{x}) \mathbf{x}^{T}+\mathbf{x} J(\mathbf{x})^{T} J(\mathbf{x}) \mathbf{x}^{T} .
\end{aligned}
$$

Observe that $J(\mathbf{x})^{T} J(\mathbf{x})=\|J(\mathbf{x})\|_{2}^{2}$ is scalar and commutes with any matrix, hence $\mathbf{x} J(\mathbf{x})^{T} J(\mathbf{x}) \mathbf{x}^{T}=\|J(\mathbf{x})\|_{2}^{2} \mathbf{x} \mathbf{x}^{T}$. Therefore, by taking advantage of the fact that the 
trace is a linear operator,

$$
\operatorname{tr}\left[\mathbf{x} J(\mathbf{x})^{T} J(\mathbf{x}) \mathbf{x}^{T}\right]=\|J(\mathbf{x})\|_{2}^{2} \operatorname{tr}\left(\mathbf{x} \mathbf{x}^{T}\right)=\|J(\mathbf{x})\|_{2}^{2} \operatorname{tr}\left(\mathbf{x}^{T} \mathbf{x}\right)=\|J(\mathbf{x})\|_{2}^{2}\|\mathbf{x}\|_{2}^{2} .
$$

Furthermore, since $J(\mathbf{x})^{T} A \mathbf{x}$ is scalar, $\operatorname{tr}\left[J(\mathbf{x})^{T} A \mathbf{x}\right]=J(\mathbf{x})^{T} A \mathbf{x}$ holds. As a result, one obtains

$$
\begin{aligned}
\operatorname{tr}\left[A^{T} J(\mathbf{x}) \mathbf{x}^{T}\right] & =\operatorname{tr}\left[\left(A^{T} J(\mathbf{x}) \mathbf{x}^{T}\right)^{T}\right]=\operatorname{tr}\left[\mathbf{x} J(\mathbf{x})^{T} A\right] \\
& =\operatorname{tr}\left[\mathbf{x}\left(J(\mathbf{x})^{T} A\right)\right]=\operatorname{tr}\left[\left(J(\mathbf{x})^{T} A \mathbf{x}\right)\right]=J(\mathbf{x})^{T} A \mathbf{x}
\end{aligned}
$$

and the theorem is proven.

Observe that for $\mathbf{x} \in \mathbb{R}^{n}$ such that $\|x\|_{2}=1$, one has

$$
\|\mathbf{x}\|_{2}^{2}\|J(\mathbf{x})\|_{2}^{2}-2 J(\mathbf{x})^{T} A \mathbf{x}=J(\mathbf{x})^{T}(J(\mathbf{x})-2 A \mathbf{x}) .
$$

In the implementation of the method, this latter expression will be used to avoid arithmetic multiplications.

In view of Theorem 4, Theorem 5, and (4), one finds that Problem 2 is equivalent to the following:

Problem 3. Let $A \in M_{n, n}^{+}$. Find $\mathbf{x} \in M_{n, 1}^{+}$such that $\|\mathbf{x}\|_{2}=1$ and

$$
\min _{\mathbf{y} \in M_{n, 1}^{+},\|\mathbf{y}\|_{2}=1}\left\{J(\mathbf{y})^{T}(J(\mathbf{y})-2 A \mathbf{y})\right\}=J(\mathbf{x})^{T}(J(\mathbf{x})-2 A \mathbf{x}) .
$$

The Lagrangian multiplier method can be readily used to solve Problem 2 with respect to the 1-norm or Problem 3. It is clear that if $\mathbf{x}$ is a solution of Problem 2, then $\lambda \mathbf{x}$ is a solution of Problem 1 for any $\lambda>0$.

In the next result, it is shown that the set of solutions of Problem 2 is not empty.

Theorem 6. Let $A \in M_{n, n}^{+}$. There exists $\mathbf{x} \in M_{n, 1}^{+}$such that $\|\mathbf{x}\|_{1}=1$ and

$$
\min _{\mathbf{y} \in M_{n, 1}^{+},\|\mathbf{y}\|_{1}=1}\left\|A-J(\mathbf{y}) \mathbf{y}^{T}\right\|_{\mathrm{F}}=\left\|A-J(\mathbf{x}) \mathbf{x}^{T}\right\|_{\mathrm{F}} .
$$

Proof: Let us introduce the following subsets of $\mathbb{R}^{n}$ :

$$
C=\left\{\mathbf{y} \in M_{n, 1}^{+}:\|\mathbf{y}\|_{1}=1\right\}
$$

$$
S=\left\{\mathbf{y}=\left[\begin{array}{lll}
y_{1} & \cdots & y_{n}
\end{array}\right]^{T} \in \mathbb{R}^{n}:\|\mathbf{y}\|_{1}=1 \text {, there exists } i \text { such that } y_{i}=0\right\} .
$$


Let $\varepsilon>0$ satisfy $C \backslash \cup_{\mathbf{y} \in S} B(\mathbf{y}, \varepsilon) \neq \varnothing$, where $B(\mathbf{y}, \varepsilon)$ denotes the open ball centered at $\mathbf{y} \in \mathbb{R}^{n}$ with radius $\varepsilon$. Finally, let us denote $D=C \backslash \cup_{\mathbf{y} \in S} B(\mathbf{y}, \varepsilon)$.

Observe that the minimisation Problem 2 is carried over the compact set $C$, however the function $\mathbf{y} \mapsto\left\|A-J(\mathbf{y}) \mathbf{y}^{T}\right\|_{\mathrm{F}}$ is not defined on the whole set $C$ : in fact, if $\mathbf{y} \in S$, then $J(\mathbf{y})$ is not well defined in view of some division by zero. Hence, it will necessary to manage the smaller set $D$.

Obviously $D$ is bounded because $D \subset C$. Also, $D$ is closed because $C$ is closed and $\cup_{\mathbf{y} \in S} B(\mathbf{y}, \varepsilon)$ is open (an arbitrary union of open balls). Therefore, $D$ is compact. Moreover, the function $f: D \rightarrow \mathbb{R}$ given by $f(\mathbf{y})=\left\|A-J(\mathbf{y}) \mathbf{y}^{T}\right\|_{\mathrm{F}}$ is continuous, hence there exists $\mathbf{x} \in D$ such that $\min _{\mathbf{y} \in D}\left\|A-J(\mathbf{y}) \mathbf{y}^{T}\right\|_{\mathrm{F}}=\left\|A-J(\mathbf{x}) \mathbf{x}^{T}\right\|_{\mathrm{F}}$.

Since

$$
\lim _{\mathbf{y} \rightarrow \mathbf{p}, \mathbf{y} \in C}\left\|A-J(\mathbf{y}) \mathbf{y}^{T}\right\|_{\mathrm{F}}=+\infty
$$

holds for any $\mathbf{p} \in S$,

$$
\min _{\mathbf{y} \in C}\left\|A-J(\mathbf{y}) \mathbf{y}^{T}\right\|_{\mathrm{F}}=\min _{\mathbf{y} \in D}\left\|A-J(\mathbf{y}) \mathbf{y}^{T}\right\|_{\mathrm{F}}
$$

is obtained. The proof is finished.

Problem 3 can be solved numerically using any optimisation library or integrated in some specific platform built using any of the existing computer environments. Once vector $\mathbf{x}$ is obtained, the sought consistent matrix may be readily built: $J(\mathbf{x}) \mathbf{x}^{T}$.

However, this consistent matrix may not fully reflect the original expert judgments. Experts may wish to enforce their know-how, and propose the modification of one or more entries of matrix $J(\mathbf{x}) \mathbf{x}^{T}$. In facing the problem of how to overcome inconsistency in AHP while still taking into account expert know-how, the described procedure must be integrated into a suitable tool to balance the latter with the former. The simple decision-support expert system presented in the next section integrates the straightforward optimisation procedure described in this section along with a simple method of eliciting information to achieve a compromise that produces optimal comparison matrices. 


\section{Implementing the process}

In this section, a tool developed in MatLab for implementing an iterative process to streamline the trade-off between expert know-how and synthetic consistency obtained by using the results of the previous section is presented. Figure 1 shows the GUI (guided user interface) containing the problem elements. The various criteria can be selected at the top left-hand side. The criteria matrix (top centre) is then able to accept expert judgment using Saaty's 9-point scale. This scale is presented on the right for convenience. In the second row of elements, the alternative comparison matrices for the various criteria may be introduced.

Most provided matrices will almost certainly be non-consistent, and with nonnegligible probability will not have acceptable consistency ratios. In addition to the method presented in this paper, various methods for consistency improvement are also implemented, namely NGMR, and the methods described in $[6,25,33,34]$. Any of the implemented prioritising processes, in particular, the optimisation described in this paper, can now be used to build a consistent matrix.

The matrix selected by the user is shown in the lower-central part of the GUI and subject to consistency improvement through any of the available methods. Specifically, for the prioritising method described in this paper the function fmincon (from the MatLab Optimization Toolbox) is executed to perform the optimisation process described in Problem 2. This function minimises the expression in Problem 3, while considering the constraint therein contained.

The new matrix thus generated with non-negligible probability may now be considered by the expert(s) to partially reflect their opinions and they may choose to modify some of the matrix entries. Shifting one or more entries of the matrix while preserving reciprocity will produce an inconsistent matrix, and a similar process can again be undergone in an attempt to reach a reasonable trade-off between consistency and expert know-how compliance.

The final decision may be accessed using the options in the lower right-hand area. This tool has been used to develop the decision-making process described in the paragraph below. Associated documentation can be found online at [35], and the tool is 
available from the second author on request.

\section{Application for water leakage management}

For the sake of simplicity, a problem with only two management alternatives for leakage control to achieve the stated objective of minimising water losses is considered. These alternatives are active leakage control (ALC) and passive leakage control (PLC). ALC is associated with the enforcement of a specific project and involves taking action to identify and repair leaks that have not been reported in distribution systems or individual district metered areas. On the other hand, PLC (no specific project considered) boils down to just repairing reported or evident leaks [36]. Even though this is a simplified statement of an important real-world problem, it is used to numerically exemplify the optimisation developed in Section 3. This problem is similar to another problem considered in [6]. Nevertheless, the problem is here extended to a wider range of criteria, and is solved with the method proposed in this paper which, in contrast with the method used in [6], uses only $n$ decision variables.

The criteria used in this paper to decide on the eventual alternative(s) are the following:

$\mathrm{C}_{1}$ : planning development cost and its implementation;

$\mathrm{C}_{2}$ : damage to properties and other service networks;

$\mathrm{C}_{3}$ : effects of supply disruptions (compensations, required time to re-fill the network, presence of trapped air pockets and related problems such as reduction of system capacity, transient effects, water quality impairment, etc.);

$\mathrm{C}_{4}$ : inconveniences caused by closed or restricted streets;

$\mathrm{C}_{5}$ : water extractions (benefits for aquifers, wetlands or rivers);

$\mathrm{C}_{6}$ : building of storage infrastructures (environmental and recreational impacts);

and

$\mathrm{C}_{7}$ : $\mathrm{CO}_{2}$ emissions (produced by the used energy, mainly by pumps). 
The first step involves building a suitable matrix of criteria that embodies knowhow regarding this specific problem. In this case, a decision was made about taking the point of view of the management of a supply company - OOAPAS (a public water company) in Morelia, Michoacán (Mexico). Since more criteria - some of which were unfamiliar to second level management staff - were used (compared with the problem presented in [6]), judgment from a top ranking group of experts in the company was the key interest in this application. The results correspond with the conclusions of this panel of experts, and were compiled after comprehensive discussion. As a consequence, the entries of this matrix represent the expert knowledge of the company managers. Upon evaluation and following the nine-point Saaty scale, matrix $A$ in Table 1 was produced to reflect the opinions regarding the relative importance of the seven criteria.

\begin{tabular}{c|ccccccc|} 
& $\mathrm{C}_{1}$ & $\mathrm{C}_{2}$ & $\mathrm{C}_{3}$ & $\mathrm{C}_{4}$ & $\mathrm{C}_{5}$ & $\mathrm{C}_{6}$ & $\mathrm{C}_{7}$ \\
\hline $\mathrm{C}_{1}$ & 1 & 7 & 3 & 5 & 9 & 3 & 5 \\
$\mathrm{C}_{2}$ & $1 / 7$ & 1 & 3 & 3 & 5 & 3 & 3 \\
$\mathrm{C}_{3}$ & $1 / 3$ & $1 / 3$ & 1 & $1 / 5$ & $1 / 3$ & 3 & 3 \\
$\mathrm{C}_{4}$ & $1 / 5$ & $1 / 3$ & 5 & 1 & 9 & 3 & $1 / 3$ \\
$\mathrm{C}_{5}$ & $1 / 9$ & $1 / 5$ & 3 & $1 / 9$ & 1 & 3 & $1 / 5$ \\
$\mathrm{C}_{6}$ & $1 / 3$ & $1 / 3$ & $1 / 3$ & $1 / 3$ & $1 / 3$ & 1 & $1 / 3$ \\
$\mathrm{C}_{7}$ & $1 / 5$ & $1 / 3$ & $1 / 3$ & 3 & 5 & 3 & 1 \\
\hline
\end{tabular}

Table 1: Matrix of criteria, $A$

Clearly this matrix is positive, homogeneous, and reciprocal, but not consistent. For example, $a_{12} a_{23}=7 \cdot 3 \neq a_{13}=3$. The Perron eigenvalue is $\lambda_{\max } \simeq 9.5$, which gives a consistency index $\mathrm{CI} \simeq 0.416$ and a consistency ratio $\mathrm{CR} \simeq 30.8 \%$. According to Saaty's criterion $[7,9]$, the consistency of this matrix is inadmissible. Neither does it pass the scale-independent criterion given by Monsuur, since $\lambda_{\max }>7.87$ [22].

Matrix $A$ is now used to build the problem described in Problem 2. This problem is solved by applying the optimisation processes described in Theorem 6. Starting with an initial iterate defined by the vector whose 7 components are $1 / 7$, the MatLab 


\begin{tabular}{c|ccccccc|} 
& $\mathrm{C}_{1}$ & $\mathrm{C}_{2}$ & $\mathrm{C}_{3}$ & $\mathrm{C}_{4}$ & $\mathrm{C}_{5}$ & $\mathrm{C}_{6}$ & $\mathrm{C}_{7}$ \\
\hline $\mathrm{C}_{1}$ & 1 & 2.017 & 4.418 & 1.285 & 9.714 & 4.774 & 2.713 \\
$\mathrm{C}_{2}$ & 0.496 & 1 & 2.191 & 0.637 & 4.817 & 2.367 & 1.345 \\
$\mathrm{C}_{3}$ & 0.226 & 0.456 & 1 & 0.291 & 2.199 & 1.081 & 0.614 \\
$\mathrm{C}_{4}$ & 0.778 & 1.569 & 3.438 & 1 & 7.559 & 3.715 & 2.111 \\
$\mathrm{C}_{5}$ & 0.103 & 0.208 & 0.455 & 0.132 & 1 & 0.491 & 0.279 \\
$\mathrm{C}_{6}$ & 0.209 & 0.422 & 0.925 & 0.269 & 2.035 & 1 & 0.568 \\
$\mathrm{C}_{7}$ & 0.369 & 0.743 & 1.628 & 0.474 & 3.58 & 1.76 & 1 \\
\hline
\end{tabular}

Table 2: Matrix of criteria, $B$

function fmincon produces the vector

$$
\mathbf{x}=\left[\begin{array}{lllllll}
0.29 & 0.59 & 1.30 & 0.38 & 2.86 & 1.41 & 0.80
\end{array}\right]^{T},
$$

that generates the consistent matrix given in Table 2, which, accordingly, is the closest consistent matrix to $A$ in the sense of the Frobenius norm. Following the iterative process described in Section 4, a reasonable trade-off between consistency and expert know-how compliance is eventually reached. It will be supposed that matrix $B$ in Table 2 embodies this trade-off.

For this matrix $B$, the normalised Perron eigenvector is:

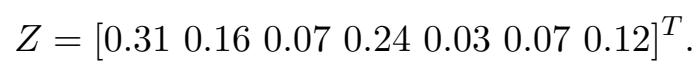

$Z$ represents the priority vector regarding the seven considered criteria. Higher values for Z's components correspond to more weighted criteria in the evaluation process; while lower values refer to criteria regarded as less important. In this case, the largest value corresponds to planning development costs and implementation. The lowest value corresponds to water extraction.

As a multi-criteria decision-making method, the AHP also uses derived composite priorities of alternatives obtained from their priorities with respect to each criterion. The simplest way to compose priorities consists of multiplying each priority of an alternative by the priority of its corresponding criterion and adding through all the criteria to obtain the overall priority of that alternative [32]. Thus, the next step 


\begin{tabular}{||cc|c||}
\hline \hline \multicolumn{1}{|c|}{ Planning } & p.v. \\
\hline 1 & 9 & 0.9 \\
$1 / 9$ & 1 & 0.1 \\
\hline \hline
\end{tabular}

\begin{tabular}{||cc|c||}
\hline \hline Damage & p.v. \\
\hline 1 & $1 / 3$ & 0.25 \\
3 & 1 & 0.75 \\
\hline
\end{tabular}

\begin{tabular}{|c|c|}
\hline Supply & p.v. \\
\hline $1 \quad 1 / 5$ & 0.17 \\
\hline 5 & 0.83 \\
\hline
\end{tabular}

\begin{tabular}{|c|c|}
\hline Closed & p.v. \\
\hline $1 \quad 1 / 3$ & 0.25 \\
\hline 3 & 0.75 \\
\hline
\end{tabular}

\begin{tabular}{||lc|c||}
\hline \hline Extraction & p.v. \\
\hline 1 & $1 / 3$ & 0.25 \\
3 & 1 & 075 \\
\hline \hline
\end{tabular}

\begin{tabular}{||lc|c||}
\hline \hline Reservoir & p.v. \\
\hline 1 & $1 / 5$ & 0.17 \\
5 & 1 & 0.83 \\
\hline \hline
\end{tabular}

\begin{tabular}{||cc|c||}
\hline \hline \multicolumn{2}{|c|}{$\mathrm{CO}_{2}$} & p.v. \\
\hline 1 & 9 & 0.90 \\
$1 / 9$ & 1 & 0.10 \\
\hline \hline
\end{tabular}

Table 3: Matrices of alternative comparisons according to the seven established criteria and their corresponding priority vectors

is to obtain vectors of priorities for our two alternatives, namely ALC and PLC, for each criterion. These vectors will reflect the weight, or relative importance, of each alternative for each criterion [8]. Calculation of these priority vectors is straightforward since the seven matrices are $2 \times 2$. In fact, as said, reciprocal $2 \times 2$ matrices are always consistent. As a result, any column of any such matrices is a principal eigenvector (corresponding to $\lambda_{\max }=2$ ). Consequently, normalisation of any of these columns directly gives the sought priority vector. The seven priority vectors are given in Table 3 for any of the alternative comparison matrices also obtained during the workshop with the panel of experts. Elements $(1,2)$ of each matrix corresponds to the attributed importance of ALC over PLC, regarding each displayed criterion.

Finally, the main target is accomplished by aggregating these scores - a synthesis of priorities to determine the best decision. A decision score is computed for any alternative by multiplying its priority value times the priority of any criterion and summing through all the criteria: 


$$
W=\left[\begin{array}{lllllll}
0.90 & 0.25 & 0.17 & 0.25 & 0.25 & 0.17 & 0.90 \\
0.10 & 0.75 & 0.83 & 0.75 & 0.75 & 0.83 & 0.10
\end{array}\right]\left[\begin{array}{c}
0.31 \\
0.16 \\
0.07 \\
0.24 \\
0.03 \\
0.07 \\
0.12
\end{array}\right]=\left[\begin{array}{l}
0.52 \\
0.48
\end{array}\right] .
$$

The largest coordinate of $W$ will be associated with 'the best alternative' and the lowest with 'the worst alternative' [15].

As a consequence, in this specific problem there is no clear preference for any alternative, although the ALC policy is slightly preferred over PLC. It is remarkable that the first impression of the co-author who acted as a facilitator, and the priority vector obtained before optimisation, pointed towards a slightly greater difference between both alternatives, and showed an a priori clear inclination for ALC over PLC by the decision-maker. Nevertheless, the economic aspects become relevant when consistency is enforced, and the stark reality is that the planning development cost and its implementation $\left(\mathrm{C}_{1}\right)$ that a water utility may incur for ALC, as much as PLC, play a leading role in the decision. A clear interpretation can be inferred taking into account the specific location of the study - where the economic conditions clearly prevail over other criteria. The second factor influencing this decision must be attributed to potential inconveniences caused by closed or restricted streets. The interesting aspect regarding the application of AHP is indeed the inclusion of social costs in decision-making. In a similar way, environmental costs, and all the externalities and normal costs for leakage management can also be included.

\section{Conclusions}

Decision making in engineering is becoming progressively more complex. Problems involve more decision variables, sophisticated constraints, and conflicting objectives; and in many cases problems are pervaded by uncertainty and subjectivity. Although 
pairwise comparisons, as in AHP, have been seen as an effective way for eliciting qualitative data, a major drawback is that when dealing with intangibles, judgments are rarely consistent - no matter how much effort is made - unless forced in some artificial manner. In this paper, after revising a number of spectral properties of the so-called comparison matrices, it is shown that consistency can be economically achieved by minimising the distance between the original matrix and a rank-one matrix built from a single vector. The main property is given by Theorem 1 , which states that a positive matrix $A$ is consistent if and only if there is a vector $\mathbf{x}$ such that $A=J(\mathbf{x}) \mathbf{x}^{T}$, where $J(\mathbf{x})$ is the vector of the reciprocal coordinates of $\mathbf{x}$. A standard minimisation procedure involving the well-behaved Frobenius matrix norm provides the sought solution, as proven by Theorem 6 . Remember that the Frobenius matrix norm minimises the root mean square variance of the differences between the judgment coefficients and the ratios between the related components of the priority vector. In addition, it is worth noting that, in contrast to other published optimisation approaches, the procedure presented here involves a much reduced number of decision variables, specifically a number equal to the order of the matrix.

This optimisation process has been implemented within an iterative feedback process that achieves an acceptable level of consistency while complying to some degree with expert preferences. As a consequence, it can be used as a decision support system for streamlining the trade-off between expert reliability and synthetic consistency.

The obtained results have been applied to a simplified version of a complex problem in engineering: the selection of a suitable policy to manage a water supply network to avoid water losses - a worrying and crucial issue in water management. The interest of the application herein presented is that it goes beyond the classical evaluation of the water losses from a mere economic point of view. The results show that the inclusion of social and environmental costs point slightly in the direction of ALC as the best alternative in leakage control. Even though in this specific case, the economic aspects remain the most important factors, a clear upsurge of other aspects can be observed. A clear interpretation can be inferred taking into account the location of the study, namely, that economic conditions clearly prevail over other criteria. Nevertheless, it can also be stated that water supply managers and authorities should, accordingly, 
shift direction from purely economic policies towards new policies that include social and environmental dimensions.

The main objective of the paper is to provide an optimisation method for comparison matrix prioritisation. The $7 \times 7$ criteria matrix in Table 1 is used to exemplify the approach. For the sake of simplicity only two alternatives are considered. In effect, reciprocal $2 \times 2$ matrices are always consistent, so the alternative matrices did not need consistency improvement. Nevertheless, considering a wider range of alternatives is straightforward, and the same consistency improvement method could be applied to all the alternative matrices.

Finally, the proposed method can be easily applied or extended to the challenging case of group decision making [24, 37]. Various approaches can be devised. One could consider individual comparison matrices, then obtain the matrix of the geometric mean of the expert judgments and apply the process described in this paper to this matrix. Another alternative could be the individual application of the process to the expert matrices and, finally, the use some type of voting system to produce the final priority vector. Another approach could minimise some aggregate value of distances between individual matrices and the matrix $J(\mathbf{x}) \mathbf{x}^{T}$, where $\mathbf{x}$ is the sought priority vector. Another possibility worth exploring would compute interval bounds for the expert judgment and then include these bounds into the optimisation problem as constraints to be satisfied. In the case of many decision makers, various voting systems could be considered with different purposes, such as eliminating outliers, aggregating values, etc.

\section{Acknowledgements}

This work has been performed under the support of project IDAWAS, DPI2009-11591 of the Direcciónn General de Investigación del Ministerio de Educación y Ciencia (Spain) and ACOMP/2011/188 of the Conselleria de Educación de la Generalitat Valenciana. The first author was supported by Spanish project MTM2010-18539. The third author is also indebted to the Universitat Politècnica de Valéncia for the sabbatical leave granted during the first semester of 2011. The use of English in this paper 
was revised by John Rawlins.

\section{References}

[1] Tabesh M, Asadiyani Yekta AH, Burrows R. An Integrated Model to Evaluate Losses in Water Distribution Systems. Water Resources Management, 23, (2009) 477-492.

[2] Nicolini M, Giacomello C, Deb K. (2011) Calibration and Optimal Leakage Management for a Real Water Distribution Network. Journal of Water Resources, Planning and Management (ASCE), 137 (2011) 134-142.

[3] Puust R, Kapelan Z, Savic DA, Koppel T. A review of methods for leakage management in pipe networks. Urban Water Journal, 7 (2010) 25-45.

[4] Alvisi S, Franchini M. Multiobjective Optimization of Rehabilitation and Leakage Detection Scheduling in Water Distribution Systems. Journal of Water Resources, Planning and Management (ASCE), 135 (2009) 426-439.

[5] Trow S, Farley M. Developing a strategy for leakage management in water distribution systems Water Science and Technology: Water Supply, 4 (2004) 149-168.

[6] Delgado-Galván X, Pérez-García R, Izquierdo J, Mora-Rodríguez J. Analytic Hierarchy Process for Assessing Externalities in Water Leakage Management. Math. Comput. Modelling, 52 (2010) 1194-1202.

[7] Saaty TL. Relative Measurement and Its Generalization in Decision Making. Why Pairwise Comparisons are Central in Mathematics for the Measurement of Intangible Factors. The Analytic Hierarchy/Network Process, Rev. R. Acad. Cienc. Exactas Fís. Nat. Ser. A Mat. 2008; 102(2): 251-318.

[8] Ho W. Integrated analytic hierarchy process and its applications - A literature review. European J. Oper. Res. (2008); 186: 211-228.

[9] Saaty TL. A scaling method for priorities in hierarchical structures. J. Math. Psych. (1977); 15: 234-281. 
[10] Brugarolas M, Martínez-Carrasco L, Bernabeu R, Martínez-Poveda A. A contingent valuation analysis to determine profitability of establishing local organic wine markets in Spain, Renewable Agriculture and Food Systems 25 (2010) 35-44.

[11] Reid E, Keith B, Comparing land use forecasting methods: expert panel versus spatial interaction model, Journal of the American Planning Association 75 (2009) 343-357.

[12] Elmer F, Seifert I, Kreibich H, Thieken AH. A DELPHI method expert survey to derive standards for flood damage data collection. Risk Analysis 30 (2010) 107-124.

[13] Dong Y, Xu Y, Li H, Dai M. A comparative study of the numerical scales and the prioritization methods in AHP. European J. Oper. Res. (2008); 186: 229-242.

[14] Saaty TL. The Analytic Hierarchy Process. McGraw-Hill. 1980.

[15] Srdjevic B. Linking analytic hierarchy process and social choice methods to support group decision-making in water management. Decision Support Systems (2007); 42: 2261-2273.

[16] Chu ATW, Kalaba RE, Springarn K. A comparison of two methods for determining the weights of belonging to fuzzy sets. J. Optim. Theory Appl. (1979); 4: 531-541, 1979.

[17] Crawford G, Williams C. A note on the analysis of subjective judgement matrices. J. Math. Psych. (1985); 29: 387-405.

[18] Finan JS, Hurley WJ. The analytic hierarchy process: Does adjusting a pairwise comparison matrix to improve the consistency ratio help? Comput. Oper. Res. (1997); 24: 749-755.

[19] Yuen KKF. Analytic Hierarchy Prioritization Process in the AHP Applications Development: A Prioritization Operator Selection Approach. Applied Soft Computing 10 (2010) 975-989.

[20] Stewart G, Sun JG. Matrix Perturbation Theory, Academic Press, 1990. 
[21] Meyer CD. Matrix Analysis and Applied Linear Algebra, SIAM, 2000.

[22] Monsuur H. An intrinsic consistency threshold for reciprocal matrices. European J. Oper. Res. 96 (1996) 387-391.

[23] Benítez J, Delgado-Galván X, Izquierdo J, Pérez-García R. Consistent Matrices and Consistency Improvement in Decision-Making Processes. in B.H.V. Topping, J.M. Adam, F.J. Pallarés, R. Bru, M.L. Romero, (Editors), Proceedings of the Seventh International Conference on Engineering Computational Technology, Civil-Comp Press, Stirlingshire, UK, Paper 21, 2010. doi:10.4203/ccp.94.21

[24] Chandran B, Golden B, Wasil E. Linear programming models for estimating weights in the analytic hierarchy process. Comput. Oper. Res. 32 (2005) 22352254 .

[25] Aznar J, Guijarro F. Nuevos métodos de valoración, Modelos Multicriterio. Universidad Politècnica de Valencia, Spain, 2008.

[26] Ji P, Jiang R., Scale transitivity in the AHP. Journal of the Operational Research Society 54 (2003) 896-905.

[27] Lin CC. An enhanced goal programming method for generating priority vectors. J. Oper. Res. Soc. 57 (2006) 1491-1496.

[28] Barzilai J. Deriving weights from pairwise comparison matrices. J. Oper. Res. Soc. 48 (1997) 1226-1232.

[29] Choo EU, Wedley WC. A common framework for deriving preference values from pairwise comparison matrices. Comput. Oper. Res. 31 (2004) 893-908.

[30] Aguarón J, Moreno-Jiménez JM. The geometric consistency index: approximated thresholds. European J. Oper. Res. 147 (2003) 137-145.

[31] Mikhailov L. A fuzzy programming method for deriving priorities in the analytic hierarchy process. J. Oper. Res. Soc. 51 (2000) 341-349.

[32] Saaty TL. Decision-making with the AHP: Why is the principal eigenvector necessary. European J. Oper. Res. 145 (2003) 85-91. 
[33] Benítez J, Delgado-Galván X, Gutiérrez JA, Izquierdo J. Balancing consistency and expert judgment in AHP. Math. Comput. Model. 54 (2011) 1785-1790.

[34] Benítez J, Delgado-Galván X, Izquierdo J, Pérez-García R. Achieving matrix consistency in AHP through linearization. Appl. Math. Model. 35 (2011) 4449-4457.

[35] Delgado-Galván, XV. Aplicación del método de jerarquías analíticas (AHP) a la gestión de pérdidas de agua en redes de abastecimiento. $\mathrm{PhD}$ dissertation, Valencia, 2011, http://riunet.upv.es/handle/10251/11238

[36] Farley M, Trow S. Losses in Water Distribution Networks. A practitioner's guide to assessment, monitoring and control. IWA publishing, 2003.

[37] Condon E, Golden B, Wasil E. Visualizing group decisions in the analytic hierarchy process. Computers and Operations Research 30 (2003), 1435-45. 